\title{
O Impacto do Programa Preventivo-Educativo da Unidade de Saúde da Família Viçosa na Saúde Bucal de Escolares
}

The Impact of the Preventive and Educational Program of the Viçosa Family Health Unit in Oral Health of Schoolchildren

\author{
Nathália Maria Lopes dos Santos ${ }^{1}$, Caroline Maders ${ }^{2}$, Vânia Maria Aita de Lemos ${ }^{3}$, Léa Fabiana Baron Ziembowicz ${ }^{4}$
}

\section{Abstract}

The Family Health Program was created by the Brazilian Health Ministry in 1994, prioritizing health protection and promotion. Prevention has been the focus of dentistry, representing a shift from an exclusively restorative approach. The aim of this study is to evaluate the impact of a preventive and educational program developed by the oral health team of the Viçosa Family Health Service at Thereza Noronha Carvalho School. The preventive and educational program has been carried out since 2001, consisting of weekly supervised brushing in students from first to eighth grade. This is a longitudinal study, with secondary data retrieved from clinical examinations carried out by the oral health team in May and September of 2010 . The following variables were collected: visible plaque, gingivitis and active white spot lesions, in the present Survey of Oral Health in Schools of Municipal Health. The differences in respect to the variables of interest between the two periods of evaluation were checked using Chi-square tests. One hundred and fifty-three children were evaluated during may and 146 children were evaluated in the follow up during September, 2010. Children enrolled in $1^{\text {st }}, 2^{\text {nd }}$ and $3^{\text {rd }}$ years were evaluated. The mean age of the sample was 7.59. There was a significant difference between the baseline and follow up examinations in respect to visible plaque [135 (88.24\%) vs. $9(6.16 \%), p=0.000]$, gingivitis [143 $(93.46 \%)$ vs. $57(39.04 \%), p=0.000]$ and active white spot lesion [81 $(52,94 \%)$ vs. $18(12,33 \%), p=0.000]$. The findings suggest that a preventive-educative intervention was effective in the improvement of oral hygiene and in the reduction of the frequency of active white spot lesions in the studied children.

Keywords: Health education; Family health; Oral health.

\section{Resumo}

O Programa Saúde da Família (PSF) foi criado pelo Ministério da Saúde em 1994, priorizando as ações de proteção e promoção à saúde. A prevenção tem sido também o enfoque da Odontologia que tem deixado de priorizar apenas o tratamento. $O$ presente estudo tem como objetivo avaliar o impacto do programa preventivo-educativo realizado pela Equipe de Saúde Bucal (ESB) da Estratégia de Saúde da Família (ESF) Viçosa na Escola Thereza Noronha Carvalho. O programa é realizado nesta escola desde 2001, e consiste na realização de escovação supervisionada semanal em alunos de $1^{\circ}$ a $8^{\circ}$ ano do Ensino Fundamental. $O$ estudo é caracterizado como longitudinal, com dados secundários coletados a partir de exames clínicos realizados pela ESB em dois momentos, março e setembro de 2010. As variáveis analisadas foram placa visível, gengivite e mancha branca ativa, presentes no Levantamento de Saúde Bucal nas Escolas da Secretaria Municipal da Saúde. As diferenças entre as variáveis de interesse nos dois períodos de avaliação foram verificadas por meio do teste Quiquadrado. Foram avaliadas 153 crianças em março e 146 crianças em setembro de 2010 , pertencentes aos $1^{\circ} \mathrm{s}, 2^{\circ} \mathrm{s}$ e $3^{\circ} \mathrm{s}$ anos, com média de idade de 7,59 anos. Houve diferença significativa entre 0 primeiro e o segundo exames em relação às variáveis presença de placa visível [135 (88,24\%) vs. $9(6,16 \%), p=0,000]$, gengivite [143 $(93,46 \%)$ vs. $57(39,04 \%), p=0,000]$ e mancha branca ativa [81 $(52,94 \%)$ vs. $18(12,33 \%), p=0,000]$. Os achados sugerem que a abordagem preventivo-educativa foi efetiva na melhora da higiene bucal e na redução da freqüência de mancha branca ativa na amostra estudada.

Palavras-chave: Educação em saúde; Saúde da família; Saúde bucal.

${ }^{1}$ Cirurgiã-Dentista, graduada pela Universidade Federal do Rio Grande do Sul UFRGS. Residente de Saúde da Família e Comunidade do Grupo Hospitalar Conceição - GHC

${ }^{2}$ Cirurgiã-Dentista, graduada pela Universidade Federal do Rio Grande do Sul UFRGS

${ }^{3}$ Professora Aposentada do Departamento de Odontologia Preventiva e Social da Faculdade de Odontologia da Universidade Federal do Rio Grande do Sul UFRGS . Mestre em Odontologia Social pela UFRGS. Especialista em Ortopedia Funcional dos Maxilares pelo Conselho Federal de Odontologia - CFO. Especialista em Odontopediatria pela Universidade Federal de Santa Catarina UFSC

${ }^{4}$ Cirurgiã-Dentista da Unidade de Saúde da Família Viçosa, graduada pela Universidade Federal do Rio Grande do Sul - UFRGS

Correspondência: Nathália Maria Lopes dos Santos

Endereço: Rua Orlando Aita, 130 - CEP 91240-030, Porto Alegre - RS, Brasil Fone: (51) 9696-9269

E-mail: natacaio@terra.com.br

Data de Submissão: 27/01/2011

Data de Aceite: 06/06/2011

\section{Introdução}

A Odontologia tem se preocupado cada vez mais com a prevenção dos problemas que ocorrem na cavidade bucal e sua relação com a saúde geral. Uma vez que doença periodontal e cárie são considerados problemas de saúde pública, além desta última causar dor e redução no rendimento escolar, é de extrema importância que se trace uma estratégia na qual ocorra comprometimento do serviço básico de saúde e da escola.

O Programa de Saúde da Família (PSF) foi criado pelo Ministério da Saúde em 1994 e, ao contrário do modelo tradicional, centrado na doença e no hospital, prioriza as ações de proteção e promoção à saúde dos indivíduos e da família, tanto adultos, quanto crianças, sadios ou doentes, de forma integral e contínua (TRAD; BASTOS, 1998). Sendo assim, o PSF surgiu como uma estratégia para consolidação do Sistema Único de Saúde (SUS), seguindo os seus princípios de universalidade, descentralização, integralidade e participação da comunidade (ANDRADE; FERREIRA, 2006).

Com a publicação da Portaria Ministerial número 1.444 , em 28 de dezembro de 2000, anunciou-se oficialmente a inserção de profissionais de saúde bucal no PSF. A Equipe de Saúde Bucal (ESB), no contexto do PSF, apresenta-se como parte integrante e importante para a saúde da população. Nesse sentido, a Política Nacional de Saúde Bucal (PNSB) propõe a incorporação progressiva de ações de promoção e proteção em saúde, como fluoretação das águas de abastecimento, educação em saúde, higiene bucal supervisionada e aplicações tópicas de flúor (BRASIL, 2004; ALMEIDA; FERREIRA, 2008). 
Sabe-se que a avaliação de políticas e programas é de grande importância em saúde pública, tendo como propósito fundamental dar suporte a todo processo decisório no âmbito do Sistema de Saúde e devendo, assim, subsidiar a identificação de problemas e a reorientação de ações e serviços desenvolvidos, avaliar a incorporação de novas práticas sanitárias na rotina de profissionais e mensurar o impacto das ações implementadas pelos serviços e programas sobre o estado de saúde da população (BRASIL, 2008). Além disso, a avaliação dos programas desenvolvidos contribui para os esforços em busca de uma sociedade mais saudável e previne o desperdício de recursos com a implementação de programas ineficazes (FACCHINI et al., 2006; VAUGHAN, 2004). Estudos prévios sugerem que a efetividade de programas preventivo-educativos em saúde bucal tem efeitos positivos na melhora dos conhecimentos e das atitudes dos envolvidos. Entretanto, aqueles que objetivam reduzir os níveis de placa e melhorar a saúde gengival nem sempre são bem sucedidos e seus efeitos positivos são vistos à curto prazo (KAY; LOCKER, 1996).

O programa preventivo-educativo, o qual é realizado pela ESB Viçosa, vem sendo desenvolvido na escola desde 2001 e os dados do levantamento de saúde bucal são registrados, armazenados e encaminhados para a Secretaria Municipal de Saúde (SMS), somente com o objetivo de levantar as necessidades das crianças para posterior encaminhamento para tratamento, não sendo, até então, usados para avaliação das ações. Além disso, há ausência de pesquisas com o objetivo de avaliar o impacto de programas preventivo-educativos no âmbito da Estratégia de Saúde da Família (ESF) considerando as variáveis estudadas. Sendo assim, e tendo em vista que o tema é relevante na perspectiva da avaliação deste serviço de saúde bucal na Atenção Básica, devido à presença de ações programáticas sobre educação-prevenção em saúde bucal na PNSB, o presente trabalho visa avaliar o impacto do programa preventivo-educativo realizado na escola na saúde bucal dos escolares considerando as variáveis placa visível, gengivite e mancha branca ativa.

\section{Materiais e Métodos}

Trata-se de um estudo longitudinal. No entanto, pela possibilidade de haver muitas perdas de segmento, estas poderão comprometer a validade das associações e reduzir o poder estatístico. Assim, os dados foram analisados como duas amostras seccionais.

Foram coletados os dados relativos aos exames clínicos realizados em dois momentos, março e setembro de 2010, e incluídos no Levantamento de Saúde Bucal nas Escolas da SMS de Porto Alegre, RS.

O estudo foi realizado na ESF Viçosa, localizada no bairro Lomba do Pinheiro, parada 13, da cidade de Porto Alegre, Rio Grande do Sul, Brasil, que abrange um território com 927 famílias e 3708 pessoas cadastradas. E uma Unidade de Saúde integrante da ESF de Porto Alegre, composta de uma Equipe de Saúde da Família e uma ESB. Esta é composta por uma CirurgiãDentista (CD), uma Técnica em Saúde Bucal (TSB) e uma Auxiliar em Saúde Bucal (ASB). Os dados foram coletados em crianças de $1^{\circ} \mathrm{S}$ a $3^{\circ} \mathrm{S}$ anos da Escola Estadual de Ensino Fundamental Professora Thereza Noronha Carvalho, na qual é desenvolvido um programa preventivo-educativo em saúde bucal desde 2001, pela ESB da ESF Lomba do Pinheiro e que, a partir de 2007, passou a ser desenvolvido pela ESB da ESF Viçosa.

O programa consiste na orientação de higiene bucal semanal através de escovação supervisionada realizada no banheiro da escola pelo pessoal técnico, já equipado com um espelho grande e colocado na altura das crianças. O dentifrício fluoretado e as escovas dentais utilizadas são fornecidos pela ESB, sendo que estas ficam guardadas com os professores de cada turma. Além disso, durante a escovação supervisionada, os alunos são instruídos quanto aos problemas bucais e a importância da higiene na prevenção dos mesmos, a fim de conscientizá-los sobre como manter sua saúde bucal.

Todos os escolares participam do programa e, além disso, os alunos de $1^{\circ} \mathrm{S}$ a $3^{\circ} \mathrm{S}$ anos são examinados para levantamento das necessidades e posterior tratamento para os escolares residentes na área de abrangência da ESF Viçosa ou encaminhamento às Unidades de Saúde dos escolares que não pertencem à área adscrita. O levantamento das necessidades de saúde bucal é realizado no consultório odontológico da ESF, no qual as crianças entram individualmente, após serem chamadas. As crianças passam por uma escovação supervisionada previamente à realização do exame e, para tanto, trazem consigo suas escovas de dentes. O exame é feito com o auxilio de odontoscópio, seringa tríplice e iluminação direta através do refletor, sendo as anotações realizadas na ficha do Levantamento de Saúde Bucal nas escolas da SMS (PORTO ALEGRE, 2010).

A população a ser estudada foi composta por 271 crianças, pertencentes aos $1^{\circ} \mathrm{S}, 2^{\circ} \mathrm{S}$ e $3^{\circ} \mathrm{s}$ anos do Ensino Fundamental da referida escola, com idade entre 6 e 17 anos, que foram examinadas em março deste ano e foram submetidas a novo exame em setembro pela ESB, que desenvolve o programa preventivo-educativo na escola. Previamente, para o desenvolvimento do mesmo, as crianças são devidamente autorizadas pelos pais ou responsáveis, tanto para os exames clínicos, como para as ações preventivo-educativas desenvolvidas pela ESB. Foram excluídas do programa as crianças que não tenham recebido a autorização pelos pais ou responsáveis para participar do mesmo. Desta forma, somente participaram 153 crianças no primeiro exame e 146 no segundo.

Como critérios de inclusão, foram analisados os dados do exame clínico de todos os alunos matriculados nos $1^{\circ} \mathrm{S}, 2^{\circ} \mathrm{S}$ e $3^{\circ} \mathrm{s}$ anos do Ensino Fundamental da escola, coletados pela ESB e incluídos no instrumento da SMS/ Prefeitura Municipal de Saúde, denominado Levantamento de Saúde Bucal nas Escolas.

O exame inicial dos escolares foi realizado, pela CD da ESB, em março deste ano, quando da volta às aulas e previamente ao programa a ser desenvolvido na escola. Após 6 meses (em setembro), a CD realizou outro exame clínico nos escolares, utilizando os indicadores do instrumento de Levantamento Epidemiológico de Saúde Bucal. Não houve treinamento dos examinadores, pois o trabalho foi realizado no serviço e busca avaliar a efetividade de uma intervenção realizada sistematicamente pelo mesmo. Além disso, já havia iniciado a intervenção quando o estudo foi planejado.

Os dados secundários utilizados nesta pesquisa foram os que estão contidos no instrumento acima (considerando os dois momentos do preenchimento do mesmo pela ESB) e relacionados aos desfechos placa visível, gengivite e/ou sangramento gengival e mancha branca ativa. Os critérios e códigos para o preenchimento do formulário pela ESB relacionados a estas variáveis são, considerando todos os dentes presentes:

Placa visível: critério para ausência de evento é Zero (0) e presença é Um (1).

Gengivite e/ou sangramento gengival em três ou mais dentes também é Zero (0) para ausência e Um (1) para presença.

Mancha branca ativa (rugosidade, superfície desmineralizada): critério para ausência de evento é Zero (0) e presença é Um (1)

As variáveis foram mensuradas através de exame clínico, utilizando-se espelho bucal plano e seringa tríplice, com iluminação do refletor, buscando-se observar sua presença ou ausência, somente. Dentro do conhecimento existente, não há documentos escritos caracterizando detalhes metodológicos.

Optou-se em analisar, nesta pesquisa, somente estas variáveis, pois entende-se que são as mesmas que possibilitam a avaliação do impacto do programa preventivo-educativo desenvolvido na escola.

O desenho da pesquisa, igualmente, vai levar em conta outra variável relacionada ao desenvolvimento do programa. Desde o início do programa, sabe-se, por observação da ESB, da diretora 
da escola e por relato dos alunos, que um grupo de professores tem incentivado a escovação diária com os alunos, fazendo com que os mesmos escovem os dentes todos os dias, após o recreio. Um outro grupo de professores, por opção própria, não incentiva a escovação diária com seus alunos. Pretende-se, assim, verificar se existem diferenças entre os dois grupos de alunos, através da análise dos dados secundários, separando-se os alunos nos dois grupos relacionados a este fator, previamente à análise dos dados. Sendo assim, o grupo $A$ recebe incentivo dos professores, enquanto que o grupo $B$ não recebe incentivo dos professores para realização da escovação diária.

Os dados foram analisados de forma seccional, calculando-se as prevalências dos desfechos em estudo para os meses de março e setembro de 2010. Para isso, foi elaborada uma tabela no software Excel 7.0, versão Windows Office 2007, para posteriormente organização de uma base de dados no programa Epi-Info 3.4.1., versão para Windows. A análise estatística dos dados foi realizada por meio do teste Qui-quadrado $\left(\left(x^{2} \quad(\alpha=5 \%)\right.\right.$ para variáveis categóricas) através do software Stata.

\section{Resultados}

Foram avaliadas neste estudo 153 crianças em março e 146 crianças em setembro de 2010 , pertencentes aos $1^{\circ} \mathrm{S}, 2^{\circ} \mathrm{S}$ e $3^{\circ} \mathrm{S}$ anos do Ensino Fundamental da Escola Thereza Noronha Carvalho, examinadas pela ESB da ESF Viçosa, que desenvolve o programa preventivo-educativo. Das 171 crianças autorizadas a participar do programa no mês de março, 153 foram avaliadas, 72 $(42,10 \%)$ eram do sexo feminino e $81(47,37 \%)$ eram do sexo masculino, com 18 ausentes no dia do exame. No segundo exame, realizado em setembro, das 186 crianças autorizadas, 146 foram examinadas, $71(38,17 \%)$ eram do sexo feminino e $75(40,32 \%)$ eram do sexo masculino com 40 ausentes $(21,51 \%)$ no dia do exame. A idade das crianças variou entre 5 e 17 anos, com uma média de 7,59 anos. A discrepância da amostra obtida nos dois exames com relação ao total de 271 crianças matriculadas deve-se ao fato de que muitos pais/responsáveis não autorizaram a participação de seus filhos neste programa que é desenvolvido na escola.

Houve diferença significativa entre o primeiro e o segundo exames em relação às seguintes variáveis: presença de placa $[135(88,24 \%)$ vs. $9(6,16 \%), p=0,000]$, gengivite [143 $(93,46 \%)$ vs. $57(39,04 \%), p=0,000]$ e mancha branca ativa [81 $(52,94 \%)$ vs. $18(12,33 \%), p=0,000]$, conforme pode ser visto na Tabela 1. Decréscimos significativos foram observados em todas as variáveis avaliadas, sendo eles $93 \%$ para placa visível, $58 \%$ para gengivite e $76 \%$ para mancha branca ativa.

Tabela 1. Distribuição de frequência das variáveis de saúde bucal nos meses de março e setembro na Escola Estadual de Ensino Fundamental Thereza Noronha Carvalho.

\begin{tabular}{llll}
\hline VARIÁVEIS & MARÇO & SETEMBRO & TOTAL \\
\hline \multirow{2}{*}{ PLACA VISÍVEL } & $\mathrm{n}=135$ & $\mathrm{n}=9$ & $\mathrm{n}=144$ \\
& $88.24 \%$ & $6.16 \%$ & $48.16 \%$ \\
& & & $p<0.001$ \\
GENGIVITE & $\mathrm{n}=143$ & $\mathrm{n}=57$ & $\mathrm{n}=200$ \\
& $93.46 \%$ & $39.04 \%$ & $66.89 \%$ \\
& & & $p<0.001$ \\
MANCHA BRANCA ATIVA & $\mathrm{n}=81$ & $\mathrm{n}=18$ & $\mathrm{n}=99$ \\
& $52.94 \%$ & $12.33 \%$ & $33.11 \%$ \\
& & & $p<0.001$ \\
\hline
\end{tabular}

Em relação às séries escolares, há de se ressaltar que os resultados foram estatisticamente significantes apenas para a variável placa visível. No primeiro exame $(p=0,032), \quad 56(96,55 \%)$ crianças dos $1^{\circ} \mathrm{S}$ anos apresentaram placa visível, bem como 42 $(85,71 \%)$ dos $2^{\circ}$ s anos e $37(80,43 \%)$ dos $3^{\circ}$ s anos. Em contrapartida, no segundo exame $(p=0,039), 1(1,67 \%)$ criança do $1^{\circ}$ ano apresentou placa visível, $2(4,76 \%)$ dos $2^{\circ}$ s anos e $6(13,64$ 5) dos $3^{\circ}$ s anos
O fato de alguns professores incentivarem as crianças a escovarem os dentes após os lanches da escola e outros não tem relação com a presença das variáveis avaliadas. No entanto, somente a variável placa visível é estatisticamente significante $(p=0,003)$ e no primeiro exame. As demais variáveis não apresentaram diferenças estatisticamente significantes, como pode ser visto na Tabela 2.

Tabela 2. Comparação das frequências das variáveis de saúde bucal em março e setembro, entre aqueles que receberam incentivo dos professores e aqueles que não foram incentivados.

\begin{tabular}{|c|c|c|c|c|c|c|}
\hline \multirow{2}{*}{ VARIÁVEIS } & \multicolumn{2}{|c|}{ SEM INCENTIVO } & \multicolumn{2}{|c|}{ COM INCENTIVO } & \multicolumn{2}{|l|}{ TOTAL } \\
\hline & MARÇO & SETEMBRO & MARÇO & SETEMBRO & MARÇO & SETEMBRO \\
\hline $\begin{array}{l}\text { PLACA } \\
\text { VISÍVEL }\end{array}$ & $\begin{array}{l}n=65 \\
97.01 \%\end{array}$ & $\begin{array}{l}\mathrm{n}=2 \\
3.64 \%\end{array}$ & $\begin{array}{l}\mathrm{n}=70 \\
81.40 \%\end{array}$ & $\begin{array}{l}n=7 \\
7.69 \%\end{array}$ & $\begin{array}{l}\mathrm{n}=135 \\
88.24 \% \\
P=0.003\end{array}$ & $\begin{array}{l}\mathrm{n}=9 \\
6.16 \% \\
P=0.323\end{array}$ \\
\hline GENGIVITE & $\begin{array}{l}\mathrm{n}=65 \\
97.01 \%\end{array}$ & $\begin{array}{l}n=24 \\
43.64 \%\end{array}$ & $\begin{array}{l}n=78 \\
90.70 \%\end{array}$ & $\begin{array}{l}n=33 \\
36.26 \%\end{array}$ & $\begin{array}{l}\mathrm{n}=143 \\
93.46 \% \\
P=0.117\end{array}$ & $\begin{array}{l}\mathrm{n}=57 \\
39.04 \% \\
P=0.376\end{array}$ \\
\hline $\begin{array}{l}\text { MANCHA } \\
\text { BRANCA } \\
\text { ATIVA }\end{array}$ & $\begin{array}{l}n=36 \\
53.73 \%\end{array}$ & $\begin{array}{l}n=10 \\
18.18 \%\end{array}$ & $\begin{array}{l}\mathrm{n}=45 \\
52.33 \%\end{array}$ & $\begin{array}{l}n=8 \\
8.79 \%\end{array}$ & $\begin{array}{l}\mathrm{n}=81 \\
52.94 \% \\
P=0.863\end{array}$ & $\begin{array}{l}\mathrm{n}=18 \\
12.33 \% \\
P=0.094\end{array}$ \\
\hline
\end{tabular}

\section{Discussão}

Os resultados encontrados no estudo sugerem que o programa preventivo realizado na Escola Estadual de Ensino Fundamental Professora Thereza Noronha Carvalho foi efetivo na melhora da higiene bucal dos escolares, no período de seis meses, conforme verificado por meio da redução na frequência de crianças com presença de placa visível, gengivite e mancha branca ativa.

Estes resultados enfatizam a importância da abordagem realizada com as crianças como estratégia para prevenção em saúde bucal, uma vez que estas possuem grande capacidade de assimilar conhecimentos e aplicá-los ao longo do tempo. Conforme - Caderno de Atenção Básica Número 17, ações educativas e preventivas tais como a realização de escovação dental supervisionada podem ter como alvo crianças em idade pré-escolar e escolar, pelo impacto de medidas educativas e preventivas nessa faixa etária e pela importância da atuação na fase de formação de hábitos (BRASIL, 2008)

Um estudo realizado no Rio de Janeiro em crianças com idades entre 4 e 13 anos que participaram de um programa de saúde bucal que incluía escovação supervisionada semanal encontrou resultados semelhantes aos do presente estudo no que diz respeito à redução de placa visível e sangramento gengival (SILVEIRA; OLIVEIRA; PADILHA, 2002). Tais achados confirmam que escolares representam um grupo populacional relevante para realização de abordagens educativas baseadas na escola.

Com relação à variável mancha branca ativa, sugere-se que a diminuição de sua frequência seja decorrente do uso de dentifrício fluoretado na escovação supervisionada. Biesbrock et al. (1998) avaliaram a capacidade de reversão de cáries incipientes através da escovação dentária com dentifrício a base de fluoreto de sódio e fluoreto estanhoso, e demonstraram que os dentifrícios contendo fluoreto de sódio e estanhoso foram mais eficazes no tratamento de cáries incipientes, em relação ao grupo controle.

O fato de o programa ser desenvolvido, anualmente, pela ESB na mesma escola pertencente à área adstrita fez com que fosse criado um vínculo entre a equipe e os alunos, o que facilita a aprendizagem e faz com que haja continuidade das atividades, constante motivação e reforço da importância da higiene bucal. Vínculo ou longitudinalidade diz respeito à utilização regular de serviço de saúde pela população e o foco da equipe na população adstrita (ELIAS et al., 2006), e pressupõe a existência de uma fonte regular de atenção e seu uso ao longo do tempo (IBANEZ et al., 2006). As equipes de saúde bucal, inseridas a partir de 2001 no contexto do PSF, desempenham papel importante na 
prevenção de problemas bucais e na promoção de saúde para a população. A PNSB propõe a incorporação progressiva de ações de promoção e proteção em saúde, como fluoretação das águas de abastecimento, educação em saúde, higiene bucal supervisionada e aplicações tópicas de flúor (BRASIL, 2004; ALMEIDA; FERREIRA, 2008)

O fato de algumas turmas receberem incentivo por parte dos professores para a escovação diária após o lanche não resultou em diferenças estatisticamente significativas na prevalência das variáveis estudadas, exceto no momento do primeiro exame para a variável placa visível, que foi mais prevalente entre os alunos que não recebem incentivo dos professores. Entretanto, o primeiro exame ocorre no início do ano letivo e, assim, não se pode afirmar que esta diferença seja decorrente do incentivo dos professores.

Além disso, as turmas que recebem incentivo são compostas, em sua maioria, por crianças do sexo feminino que, em geral, demonstram maior interesse na execução das atividades e no autocuidado, o que pode também ter levado a uma diferença nos resultados. Um estudo em escolares de Goiânia-GO demonstrou que adolescentes do sexo feminino apresentaram freqüência de escovação maior do que adolescentes do sexo masculino (FREIRE; SHEIHAM; BINO, 2007), assim como outro estudo, com alunos de $7^{\underline{a}}$ série da cidade de Gravataí-RS (FREDDO et al., 2008).

No Levantamento Epidemiológico de Saúde Bucal das Escolas, ao qual pertencem os dados coletados neste estudo, a presença ou ausência de gengivite é aferida por meio de escovação profissional e posterior exame. Mariath et al. (2008) demonstraram em seu estudo que a escovação profissional é uma ferramenta útil para o diagnóstico de inflamação gengival, principalmente como indicador de saúde gengival. No que diz respeito ao diagnóstico de manchas brancas ativas, este deve ser realizado após a remoção do biofilme, que poderia "camuflar" a lesão, e após secagem da superfície dentária com uso da seringa tríplice (FEJERSKOV; KIDD, 2005), tal como é realizado no exame dos escolares.

Uma das limitações encontradas neste estudo foi o fato da variável placa visível ser avaliada após realizada escovação dos dentes das crianças. Tem-se, portanto, um viés de aferição, visto que se é realizada escovação, a placa que as crianças possuem é desorganizada ou mesmo eliminada com escova multicerdas. Logo, pode ter ocorrido distorção dos resultados com relação a esta variável, já que é esperado que a maioria que tenha escovado adequadamente os dentes não terá mais placa visível. Então, pode-se sugerir que a aferição realizada diz respeito à capacidade de remoção de biofilme da escovação realizada durante o levantamento de dados e não à remoção realizada pela criança. Outro aspecto que cabe ressaltar foram os altos os índices de placa visível no momento da primeira coleta de dados, apesar do programa existir desde 2001 e desde 2007 estar sendo desenvolvido pela ESB da ESF Viçosa. Isto pode ter acontecido em virtude de que, antes da coleta dos dados, as crianças estavam em período de férias escolares, no qual não recebem nenhum tipo de atenção. Além disso, houve uma interrupção do programa em um período prévio à coleta.

Outro problema encontrado está presente na metodologia. Não houve treinamento dos examinadores, pois o trabalho foi realizado no serviço e a examinadora dos exames clínicos é sempre a mesma CD (desde 2001). Como o mesmo objetivou avaliar a efetividade de uma intervenção realizada sistematicamente pelo serviço, não tinha como fazer uso do índice Kappa para concordância. Outrossim, já havia iniciado a intervenção quando o estudo foi planejado. Cabe ainda, o fato do estudo não ser totalmente cego para a intervenção, pois os examinadores sabiam apenas que as crianças de $1^{\circ} \mathrm{S}$ a $3^{\circ} \mathrm{S}$ anos participavam do estudo.

Este trabalho evidenciou a necessidade de uma atuação continuada da ESB na promoção, prevenção e educação em saúde da população adscrita da ESF Viçosa. Uma ESB completa (modalidade II) explicita a possibilidade do CD planejar, organizar, supervisionar e desenvolver uma atividade preventivo-educativa nesta comunidade, em que o TSB ou ASB irão atuar como executores, de forma que o CD possa, enquanto isso, garantir atenção ampliada e qualificada no consultório para o restante da população assistida. Assim, cabe ao CD a avaliação desta atividade, que é essencial em saúde pública, contribuindo em busca de uma sociedade mais saudável e prevenindo o desperdício de recursos com a implementação de programas ineficazes (VAUGHAN, 2004; FACCHINI et al., 2006), bem como promovendo a integralidade do cuidado. Desta forma, atuar na escola Thereza Noronha Carvalho propiciou aquisição de conhecimentos relacionados à saúde bucal para as crianças, bem como para suas famílias, as quais indiretamente recebem as informações dadas e estas acabam por serem reforçadas pela atuação da ESF neste território.

Avanços na avaliação de programas de promoção de saúde bucal são benéficos no desenvolvimento da integralidade em Atenção Primária à Saúde. A avaliação é importante como meio de avaliar a efetividade de intervenções preventivas, disseminar e divulgar exemplos de boa prática, fazer o melhor uso de recursos limitados, fornecer dados para funcionários e usuários, além de informar sobre o desenvolvimento e implementação de programas (KWAN; PETERSEN, 2004). Na Atenção Básica, a avaliação é um instrumento de gestão importante para subsidiar as tomadas de decisões e formação dos sujeitos envolvidos nos processos de gestão (BRASIL, 2008; ALMEIDA; FERREIRA, 2008).

\section{Conclusão}

A avaliação do programa preventivo-educativo desenvolvido pela ESB da ESF Viçosa, que consiste na orientação de higiene bucal semanal através de escovação supervisionada realizada na Escola Estadual de Ensino Fundamental Professora Thereza Noronha Carvalho, permite concluir que as crianças avaliadas apresentavam altos índices de placa, sangramento gengival e mancha branca ativa no momento do primeiro exame, denotando a necessidade de uma abordagem preventivo-educativa para alteração destes parâmetros. Por conseguinte, a realização de atividades que visem à educação em saúde tem nesta escola um local importante para o seu desenvolvimento, visto que esta instituição é um lugar de aquisição de conhecimentos importantes para o desenvolvimento dos indivíduos que nela estudam.

Programas educativo-preventivos em saúde devem continuar sendo realizados pela ESB da ESF Viçosa no âmbito escolar, visto que se mostraram efetivos na redução de gengivite $e$ mancha branca, além de melhorarem a capacidade das crianças em remover placa com escovação dentária. Acreditamos que tal intervenção desempenhe um papel importante no desenvolvimento do autocuidado e do despertar sobre a importância da manutenção da saúde bucal.

\section{Referências}

ALMEIDA, G.C.M.; FERREIRA, M.A.F. Saúde bucal no contexto do Programa de Saúde da Família: práticas de prevenção orientadas ao indivíduo e ao coletivo. Cad. Saúde Pública, Rio de Janeiro, v. 24, n. 9, p. 2131-2140, set. 2008.

ANDRADE, K.L.C.; FERREIRA, E.F. Avaliação da inserção da odontologia no Programa de Saúde da Família de Pompéu (MG): a satisfação do usuário. Ciênc. saúde coletiva, Rio de Janeiro, v. 11, n. 1, p. 123-130, mar. 2006.

BIESBROCK A.R. et al. Reversal of incipient and radiographic caries through the use of sodium and stannous fluoride dentifrices in a clinical trial. J. Clin. Dent., Yardley, PA, v. 9, no.1, p.5-10, 1998. 
BRASIL. Ministério da Saúde. Departamento de Atenção Básica Diretrizes da Política Nacional de Saúde Bucal. Brasília, 2004.

BRASIL. Ministério da Saúde. Departamento de Atenção Básica. Saúde bucal. Brasília, 2008. (Caderno de Atenção Básica, n. 17. Série A. Normas e Manuais Técnicos).

ELIAS, P. E. et al. Atenção Básica em Saúde: comparação entre PSF e UBS por estrato de exclusão social no município de São Paulo. Ciênc. saúde coletiva, Rio de Janeiro, v. 11, n. 3, p. 633641, 2006.

FACCHINI, L. A. et al. Desempenho do PSF no Sul e no Nordeste do Brasil: avaliação institucional e epidemiológica da Atenção Básica à Saúde. Ciênc. saúde coletiva, Rio de Janeiro, v. 11, n. 3 , p. 669-681, 2006.

FEJERSKOV, O.; KIDD, E.. Cárie dentária: a doença e seu tratamento clínico. São Paulo: Liv. Santos, 2005. Cap. 13, p.189219.

FREDDO, S. L. et al . Hábitos de higiene bucal e utilização de serviços odontológicos em escolares de uma cidade da Região Sul do Brasil. Cad. Saúde Pública, Rio de Janeiro, v. 24, n. 9, p. 1991-2000, set. 2008.

FREIRE, M. C. M.; SHEIHAM, A.; BINO, Y. A.. Hábitos de higiene bucal e fatores sociodemográficos em adolescentes. Rev. bras. epidemiol., São Paulo, v. 10, n. 4, p. 606-614, dez. 2007 .

IBANEZ, N. et al . Avaliação do desempenho da atenção básica no Estado de São Paulo. Ciênc. saúde coletiva, Rio de Janeiro, v. 11 , n. 3, p. 683-703, jul./set. 2006.

KAY, E.; LOCKER, D. Is dental health effective? A systematic review of current evidence. Community Dent. Oral Epidemiol. Copenhagen, v. 24, no. 4, p. 231-235, 1996.

KWAN, S.; PETERSEN, P.E. Evaluation of community based oral health promotion and oral disease prevention - WHO recommendations for improved evidence in public health practice. Community Dent. Health, London, v. 21, no. 4, Suppl., p. 319-329, Dec. 2004.

MARIATH, A. A. S. et al. Professional flossing as a diagnostic method for gingivitis in the primary dentition. Braz. Oral. Res., São Paulo, v. 22, no. 4, p. 316-321, Oct./Dec. 2008.

PORTO ALEGRE. Secretaria Municipal de Saúde. Levantamento de saúde bucal nas escolas. Porto Alegre, 2010.

SILVEIRA, J. L.G.C.; OLIVEIRA, V.; PADILHA, W. W. N.. Avaliação da redução do índice de placa visível e do índice de sangramento gengival em uma prática de promoção de saúde bucal com crianças. Pesqui. Odontol. Bras., São Paulo, v. 16, n. 2, p.169174, abr./jun. 2002.

TRAD, L.A.B.; BASTOS, A.C.S. O impacto sócio-cultural do Programa de Saúde da Família (PSF): uma proposta de avaliação. Cad. Saúde Pública, Rio de Janeiro, v. 14, n. 2, p. 429-435, abr./jun. 1998.

VAUGHAN, R. Evaluation and public health. Am. J. Public Health Washington, v. 94, no. 3, p. 360, Mar. 2004. 